Voix et Images

voixetimages

\title{
Questions de visibilité
}

\section{Lori Saint-Martin}

Volume 27, numéro 2 (80), hiver 2002

La sociabilité littéraire

URI : https://id.erudit.org/iderudit/290064ar

DOI : https://doi.org/10.7202/290064ar

Aller au sommaire du numéro

\section{Éditeur(s)}

Université du Québec à Montréal

\section{ISSN}

0318-9201 (imprimé)

1705-933X (numérique)

Découvrir la revue

\section{Citer cet article}

Saint-Martin, L. (2002). Questions de visibilité. Voix et Images, 27(2), 360-364. https://doi.org/10.7202/290064ar d'utilisation que vous pouvez consulter en ligne.

https://apropos.erudit.org/fr/usagers/politique-dutilisation/ 
Féminismes

\section{Questions de visibilité}

\section{Lori Saint-Martin, Université du Québec à Montréal}

Tout comme le féminin a long- tradition littéraire. On se lasse de temps été invisible ou dévalorisé dans l'écrire, mais le fait demeure. Or comla langue, les femmes ont plus sou- ment, de nos jours, les rendre visibles? vent qu'autrement été effacées de la Comment relire, et mieux encore, 
récrire la langue, la tradition, l'histoire littéraire? Ces questions se trouvent au coeur de deux ouvrages récents, l'un portant sur la féminisation linguistique, l'autre sur l'identité narrative.

L'essai de Louise-L. Larivière, Pourquoi en finir avec la féminisation linguistique ou À la recherche des mots perdus $^{1}$, est coiffé d'un titre délibérément - mais à mon sens malencontreusement - équivoque : là où on risque de comprendre qu'il faut cesser de parler de féminisation, Louise-L. Larivière affirme haut et fort qu'il faut, enfin, en faire une réalité. Écrit d'une plume alerte et parfois trempée dans le vitriol, l'ouvrage passe en revue les faiblesses du système linguistique actuel, les principaux arguments qu'on oppose à la féminisation et les grands principes au nom desquels on doit, au contraire, la faire.

Louise-L. Larivière rappelle donc en premier lieu l'absence, en français, d'un véritable générique ("homme" est un faux générique parce qu'il a aussi un sens spécifique) comme d'un genre neutre digne de ce nom (la notion de "genre non marqué", qu'on applique souvent au masculin, n'est pas fondée en linguistique puisque c'est le propre du genre d'avoir des marques du masculin ou du féminin). Les femmes sont donc absentes de la langue - "le masculin l'emporte sur le féminin", affirme-t-on encore sur tous les tons - ou encore, lorsqu'elles y figurent, sont dévalorisées. D'où la nécessité d'établir l'égalité "au moyen de noms féminins bien formés, modernes et de même valeur sémantique que les noms masculins et au moyen d'agencements qui ne donnent préséance ni à un genre ni à un autre" (p. 120). Les manières de le faire sont traitées, semble-t-il, dans un deuxième ouvrage, Comment en finir avec la féminisation linguistique ou les mots pour le dire, publié sous forme électronique aux Éditions 00h00.com.

Louise-L. Larivière démolit tour à tour, avec humour - mais aussi avec humeur -, les arguments habituellement opposés à la féminisation : possibles confusions entre état civil de la femme et fonction de son mari ("l'ambassadrice" peut être la femme de l'ambassadeur) ou entre noms de personnes et de choses ("jardinière", "causeuse", "coiffeuse"), le caractère péjoratif ou non esthétique des noms féminins, etc. Elle fustige aussi l'incohérence et les "deux poids, deux mesures". Les hommes qui exercent un métier traditionnellement féminin exigent, rappelle-t-elle, un titre bien à eux: quel homme dirait "je suis infirmière"? En France, on a créé, pour trouver le masculin de "sage-femme", une commission de terminologie qui a retenu, contre toute attente, le terme "maïeuticien", abstrait, savant et, partant, prestigieux. On peut donc faire preuve d'une grande créativité lorsque la situation l'exige: pourtant, aux dires de nombreux spécialistes, "directeur ", "médecin ", "écrivain" n'ont pas à avoir de forme féminine. Comme par hasard, pourrait-on ajouter, les métiers sans prestige particulier se féminisent obligatoirement, alors que ceux du sommet de l'échelle admettent mal le féminin: on ne dit pas "elle est boulanger", mais "madame le président " passe encore la rampe. On le voit, on n'est pas dans le domaine du linguistique, ici, mais dans celui de l'idéologie.

Au coeur de la réflexion de Louise-L. Larivière se trouve précisément la question de la logique: il 
s'agit de discuter de la féminisation d'une manière rationnelle plutôt qu'émotive, alors que les enjeux proprement linguistiques du débat ont souvent été oubliés au profit de moqueries et de cris d'outrage. Comme les linguistes féministes le rappellent depuis belle lurette (on lira Marina Yaguello, Benoîte Groult ou Louky Bersianik à ce sujet), ce n'est pas la langue elle-même qui fait obstacle au changement - elle dispose de tous les moyens nécessaires pour réaliser la féminisation - mais la société, ou du moins certains de ses éléments. Les femmes qui préfèrent encore se faire appeler "madame le directeur" suscitent autant l'ire de Louise-L. Larivière que les Académiciens qui répètent à qui mieux mieux, en feignant de se tordre les côtes de rire, que "écrivaine, ça rime avec vaine. (comme si, pour paraphraser Ducharme, on ne voyait pas "vain" dans "écrivain" tout autant qu' "Odile "dans " crocodile").

C'est sans doute cette valorisation, par ailleurs louable, de l'objectivité et du bon sens qui motive une curieuse affirmation selon laquelle "Iclet ouvrage se veut, bien sûr, polémique, mais non militant... ou si peu " (p. 12). Pourtant, lorsque Louise-L. Larivière écrit "La langue, ce n'est pas un objet esthétique ni patriotique, c'est un objet linguistique qui doit servir, entre autres, la justice sociale" (p. 21), elle milite bel et bien. On voit mal du reste comment il peut y avoir une position non partisane en la matière: soit la langue reste au service du statu quo, soit elle change pour reconnattre mais aussi pour promouvoir - l'égalité des femmes. Les deux positions sont politiques. Ainsi, qui combat pour la justice sociale et propose de mettre fin à la marginalisation d'un groupe dominé fait ouvrage militant, et il n'y a aucun mal à cela. On peut militer pour une cause objectivement bonne - l'égalité des sexes - et mettre au service de ce travail des arguments logiques plutôt que des partis pris. Ce que fait précisément ici Louise-L. Larivière. Ầ défaut de présenter de nouveaux arguments mais on ne renouvelle pas sans mal un débat aussi rebattu - , son petit essai offre une synthèse claire, accessible et intelligente de ceux qui nous sont déjà familiers. Il se clôt d'ailleurs sur une piste prometteuse: plutôt que de féminisation, peut-être faudrait-il parler de "parité linguistique"? Le débat est de nouveau ouvert...

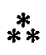

Mary Jean Green se classe parmi les plus lucides et plus perspicaces des analystes américains de la littérature québécoise. L'essai qu'elle vient de faire paraitre, Women and Narrative Identity: Rewriting the Quebec National Text ${ }^{2}$, s'inscrit dans la continuité de ses travaux passés, puisqu'une partie de son contenu est tiré des articles qu'elle a publiés sur l'écriture au féminin depuis plus de vingt ans.

Au centre du livre se trouvent les rapports paradoxaux qu'entretient l'écriture des femmes québécoises avec le projet littéraire national. Or la tradition littéraire québécoise, si elle fait aux femmes une place relativement grande (on n'a qu'à comparer avec la France où, même de nos jours, l'institution littéraire reste forte- 
ment masculine), n'en définit pas moins la littérature et ses enjeux selon des critères masculins.

Comment les femmes parviennentelles donc à s'inscrire dans cette tradition? Celles qui s'inspirent davantage du "récit identitaire québécois" ("Quebec identity narrative") accèdent le plus facilement à la consécration. Inversement, celles qui, comme Jovette Bernier et Éva Senécal dans les années 1930, produisent des œuvres en rupture avec l'idéologie et les genres dominants de leur époque, font scandale, puis sont reléguées aux oubliettes. La marge consentie à la contestation est donc réduite: trop proches du récit canonique, les écrits des femmes reproduisent en tout le statu quo socio-sexuel; trop loin de ce récit, ils seront mal reçus et négligés. Celles qui passeront à la postérité auront su naviguer avec bonheur entre le double écueil que représentent la récupération et la marginalisation, parvenant malgré tout à inscrire dans la littérature une voix et une perspective nouvelles.

Au début était donc le projet de la survivance française, le messianisme en Amérique. Le roman du XIX ${ }^{e}$ siècle est censé moraliser le peuple et lui donner le sens de son histoire. Or, à une époque où très peu de femmes écrivent au Québec - ne leur a-t-on pas vanté, à l'exclusion de tout le reste, leur noble mission maternelle? - Laure Conan, avec Angéline de Montbrun et les romans qui ont suivi, a su rendre son écriture recevable en empruntant de nombreux éléments du récit identitaire d'alors : la ferveur religieuse, la vénération du passé, la référence à des héros nationaux comme Garneau et à des héroïnes comme Marie de l'Incar- nation (sujet du reste d'une grande étude de l'abbé Casgrain, mentor littéraire de Laure Conan). L'étude que fait Mary Jean Green de ce roman montre de quelle façon Conan intègre dans le roman, entre les lignes pour une large part, une vision de femme. En effet, comme on l'a souvent montré, le roman fait entendre une voix bien plus personnelle que collective, et Angéline refuse les deux destins proposés aux femmes d'alors: le mariage et la vie religieuse. La figure de Marie de l'Incarnation autorise la méditation d'Angéline sur l'amour divin et l'amour humain, ainsi que l'expression d'une subjectivité de femme qui transite non pas par la narration impersonnelle en vogue alors, mais par le journal intime fictif. En convoquant des femmes de lettres "respectables" comme Madame de Sévigné et Eugénie de Guérin, Angéline inscrit son écriture dans la continuité plutôt que dans la rupture, dans la vertu plutôt que dans la transgression. Mais du même coup, elle crée une lignée de femmes créatrices qu'elle célèbre et qui autorise sa propre venue à l'écriture. Ainsi émergent, de l'ensemble de l'œuvre de Laure Conan, et malgré un apparent respect des conventions sociales et littéraires, un héroïsme et une passion au féminin qui refont la littérature québécoise au moment même de l'inaugurer.

Ce long résumé d'un chapitre de Women and Narrative Identity illustre la finesse des analyses, impossibles à résumer toutes ici. Ajoutons seulement que, comme Laure Conan mais autrement, Germaine Guèvremont et Gabrielle Roy auront su infléchir le genre dominant de leur époque - le roman de la terre - pour lui donner 
un visage nouveau. Germaine Guèvremont récrira l'histoire de la transmission du patrimoine en privilégiant la lignée féminine et les nouvelles options offertes aux protagonistes. Gabrielle Roy reproduira, dans un cadre urbain, nombre d'éléments du roman de la terre, dont l'intérêt pour la grosse famille et l'éloge des valeurs traditionnelles qu'incarne Rose-Anna. Habilement, les deux romancières auront su à la fois célébrer l'ordre ancien et montrer qu'il est inexorablement condamné.

$\mathrm{Si}$, dans les années 1960 , le religieux a été balayé du roman, la littérature québécoise demeure subordonnée, aux yeux de nombreux écrivains influents, à l'émergence de la Nation. Dans cette vision des choses, on ne considère généralement pas la contribution des femmes comme importante. Mary Jean Green affirme le contraire, elle qui relit, entre autres, Kamouraska d'Anne Hébert à la lumière de la théorie de la décolonisation : doublement colonisée, comme sujet de la couronne britannique et comme femme, Élisabeth se soumet à l'autorité étrangère et préfère la prison à la fuite avec George Nelson ${ }^{3}$.

Les deux derniers chapitres de Women and Narrative Identity exploitent tout autrement la notion d'identité. En effet, on ne peut plus dire que les écrivaines s'approprient des formes canoniques masculines pour s'intégrer au courant dominant. Au contraire, les créatrices des années 1970 ont instauré une nouvelle textualité en rupture avec les formes et la langue littéraires, considérées comme phallocentriques. Plus récemment, des créatrices nées au Québec (France Théoret, Francine Noël, Monique Proulx) et venues d'ailleurs (Régine Robin, Abla Farhoud, Marie-Célie Agnant), prônent une identité plus ouverte, multiple, toujours en devenir, mais, chez les écrivaines migrantes, ancrée dans une généalogie féminine intime.

L'un des grands mérites de l'essai de Mary Jean Green est de ne pas établir, pour l'écriture des femmes, une tradition parallèle - et donc mineure. Il tente au contraire de repenser le "texte québécois national" en l'élargissant pour y englober d'autres voix (y compris celles de femmes peu lues de nos jours, comme les premières journalistes du $\mathrm{XIX}^{\mathrm{e}}$ siècle). Sa lecture intéressera autant les spécialistes que les néophytes; il aura certainement une belle carrière aux États-Unis, et on peut souhaiter qu'il soit largement lu au Québec aussi. Jusqu'à un certain point, on peut parler de "deux solitudes" entre féministes littéraires québécoises et américaines: en effet, elles ne se lisent pas toujours suffisamment entre elles. Un tel ouvrage est susceptible d'ouvrir le dialogue et de jeter des ponts.

1. Louise-L. Larivière, Pourquoi en finir avec la féminisation linguistique ou $\dot{A}$ la recherche des mots perdus, Montréal, Boréal, 2000, 149 p.

2. Mary Jean Green, Women and Narrative Identity: Rewriting the Quebec National Text, Montréal/Kingston, McGill/Queen's University Press, 2001, $197 \mathrm{p}$.

3. Mary Jean Green relève la signification historique du patronyme Nelson: deux frères Nelson ont participé à l'insurrection de 1837-1838. Je me rappelle avoir lu, en revanche, qu'Anne Hébert avait d'abord appelé son personnage George Wilson, mais que les Éditions du Seuil lui ont conseillé de le changer; en effet, il existe un acteur français du même nom. 